\title{
Space astrophysical observatory «Lomonosov» : the first results
}

\author{
Mikhail Panasyuk $^{1}$ on behalf the Lomonosov - UHECR/TLE \& GRB collaborations
}

1. Skobeltsyn Institute of Nuclear Physics of Lomonosov Moscow State University, Leninskie Gory, 119991, Moscow, Russia

2. Physics department of Lomonosov Moscow State University, Leninskie Gory, 119991, Moscow, Russia

E-mail: panasyukesinp.msu.ru

\begin{abstract}
The satellite "Lomonosov" was launched on April, 28, 2016. The main objective of the experiments on board this satellite- to study of extreme physical phenomena in space. Among them are Ultra High Energy Cosmic Rays (UHECR) and cosmic transient gamma-ray bursts. For UHECR measurements on board this satellite the mirror-type ultraviolet telescope (TUS) was installed to register Extensive Air Showers (EAS) in the atmosphere initiated by UHECR. It is the world's first space experiment which can measure particles in the energy more than $5 \times 10^{19} \mathrm{eV}$, that is of the Greisen-Zatsepin-Kuzmin cut-off (GZK-cut off) possible energy band. The results of identifying candidates for EAS from UHECR have been presented. Cosmic gamma -ray bursts (GRB) on board "Lomonosov" were recorded by multiwave set of instruments: in UV, optical - visible and gamma-rays ranges by gamma-ray spectrometer (BDRG), UFFO instrument consisted of ultraviolet image telescope (SMT) with a gamma-rays detector (UBAT) and wide-field optical cameras SHOK. The first results of the analysis of observations of GRBs- their timing and power characteristics are presented.
\end{abstract}

35th International Cosmic Ray Conference - ICRC2017

10-20 July, 2017

Bexco, Busan, Korea

${ }^{1}$ Speaker 


\section{Introduction}

Among a number of scientific tasks of the project "Lomonosov" there are studies of UHECR, GRB and other cosmic gamma-ray transients . Both UHECR and GRB are directly related to extreme processes in the Universe and release of huge amounts of energy in astrophysical objects outside our Galaxy and considered as the main topics of "Astroparticle physics", will be presented in this paper.

Understanding the nature of Ultra High Energy Cosmic Rays (UHECR), energies above approximately $1 \mathrm{EeV}=10^{18} \mathrm{eV}$ (energy of possible beginning of domination of extragalactic cosmic rays (see, e.g. [1]) is one of long-standing significant mysteries of modern astrophysics closely connected with the actual fundamental problems associated with them. Among them is the problem of the existence of GZK - cutoff of energy spectrum of UHECR predicted by G. Zatsepin and V. Kuzmin [2], and K. Greizen [3], is of undoubted interest up to now.

The average slope of the all particle cosmic rays spectrum is quite steep and the flux of them varies in a wide range from 1 particle per $1 \mathrm{~m}^{2}$ in 1 second at energy of $\sim 10^{10} \mathrm{eV}$ and up to 1 particle per $1 \mathrm{~km}^{2}$ in 100 years with energy $\sim 10^{20} \mathrm{eV}$. This factor poses a difficult problem for experiments - the development of on ground installations of a large area to achieve acceptable measurement statistics. Evidently, this is especially critical for UHECR with E $>10^{18} \mathrm{eV}$. Suffices to say that the largest ever cosmic rays experiment, the Pierre Auger Observatory (Auger), which occupies an area of more than $3000 \mathrm{~km}^{2}$, registered only 146 UHECR with energies above $53 \mathrm{EeV}$ in almost eight years of operation (from May 27, 2006, to March 31, 2014) [4].

To date, there are two main problems concerning the interpretation of ground-based installations data. This is the chemical composition of UHECR and their anisotropy. Recent results of Auger and Telescope Array (TA) are significant and interesting, but there is not unambiguous interpretation of them. If the chemical composition data of these detectors are close to each-other in the presence of significant errors [5], then the data on anisotropy are significantly different. This problem evidently connected with an incomplete coverage of the celestial sphere by any existing ground arrays: the Auger in the southern hemisphere and the TA in the northern one, observe regions of the celestial sphere that only partially overlap. From another side it can be explained by naturally different locations of astrophysical objects in different hemispheres of the sky. This poses serious problems for an analysis of arrival directions (anisotropy) of UHECR, see, e.g. (see, e.g.[6,7]).

A way with attempt to overcome both difficulties was proposed by Benson and Linsley in early of 1980's $[8,9]$. They suggested to use a reflector type telescope deployed on a low-orbit satellite to register ultraviolet (UV) photons of fluorescence and Cherenkov radiation emitted by ionized molecules of nitrogen exited by charged particles of an extensive air shower (EAS) cascade born in the atmosphere by an UHECR. Shortly after the publication of the ideas of Benson \& Linsley there was a project space installation, based on the scheme of the optical telescope, consisting of Fresnel lenses with wide field of view - OWL - Orbiting Wide-angle Light collectors [10]. It was not implemented, but based on it there was another project proposed by L. Scarsi- Extreme Universe Space Laboratory (EUSO) [11]. With such orbiting wide-field telescope with a large aperture of $60^{\circ}$ it is possible to observe UHECRs at atmosphere on the area more than $10^{4} \mathrm{~km}^{2}$ at the height of the orbit of the spacecraft about 400 $\mathrm{km}$. This corresponds to the huge geometrical factor of the installation is about $2 \cdot 10^{4} \mathrm{~km}^{2}$ sr year, much more than existing for on ground installations. Then, EUSO project has been transformed in JEM - EUSO project for Japanese Experimental Module on International Space Station [12, 13]. Due to this, none of the projects suggested have been implemented yet (see, e.g., [14] for a brief review).

The other extreme phenomena in the Universe are GRBs, which are observed as short (from dozens of milliseconds up to dozens of seconds) increases of gamma ray fluxes. Discovered in 60 s years of 20th century, they are still at the cutting edge of astrophysics (see, e.g., $[15,16]$. These phenomena being the most powerful in the Universe occur not only in gamma-range, but 
also in visible optics and UV. The power of the explosion of these most bright astrophysical objects achieves $10^{51}-10^{53} \mathrm{erg} / \mathrm{s}$. GRB optical emission lasts up to several hours or even days. It can be an evidence of afterglow, which appear after a giant explosion in the external shock wave expanding in the interstellar space and stellar wind of the exploded star. Probably, it is a process of collapse of a fast-rotating very massive star to a black hole in the case of so-called longduration (more than a few seconds) bursts or merging of neutron stars in tight binary system in the case of so-called short-duration (less than a second) bursts. However, these models are under discussion and the nature of this extraordinary phenomenon is still unknown. Due unusually powerful brightness of GRBs, studying of their properties allows the researchers to explore the epoch of the early Universe, i.e. to study evolution of the stars and the stellar populations within the wide range of red shift from $\mathrm{z} \sim 0.1$ up to $\mathrm{z} \sim 15-20$, it is more than $98 \%$ of the age of our Universe. Unlike previous space experiments for observing of GRB we installed on board the satellite "Lomonosov" a complex scientific instruments for multiwave's measurement of GRB parameters for further study of their nature.

The satellite "Lomonosov" was launched on April, 28, 2016 on a sun - synchronous orbit with altitude of about $500 \mathrm{~km}$ and during more than a year transmit scientific information.

\section{UHECR's candidates search}

TUS (Tracking Ultraviolet Set-up) is the first instrument developed for measuring UHECR. It was inherited the optical scheme of the original design by Benson and Linsley but with much more modest technical parameters. TUS was first announced in 2001 [17] as a pathfinder for the future more advanced KLYPVE project [18] (today there is more advanced conception of this instrument, under the development of EUSO collaboration, named K-EUSO [19]).

The TUS detector consists of two main components: a parabolic mirror-concentrator of the Fresnel type and a square-shaped 16×16-channel photodetector in the focal plane of the mirror. The mirror has an area of about $2 \mathrm{~m}^{2}$ with a focal distance $1.5 \mathrm{~m}$. The mirror is composed of 7 hexagonal segments of equal size, made of two carbon plastic layers strengthened by a honeycomb aluminum structure. The field of view (FOV) of the detector equals $\pm 4.5^{\circ}$, which results in an approximately $80 \mathrm{~km} \times 80 \mathrm{~km}$ area at sea level. The angular resolution of one channel equals $10 \mathrm{mrad}$, which gives a $5 \mathrm{~km} \times 5 \mathrm{~km}$ area (see [20] for more details).

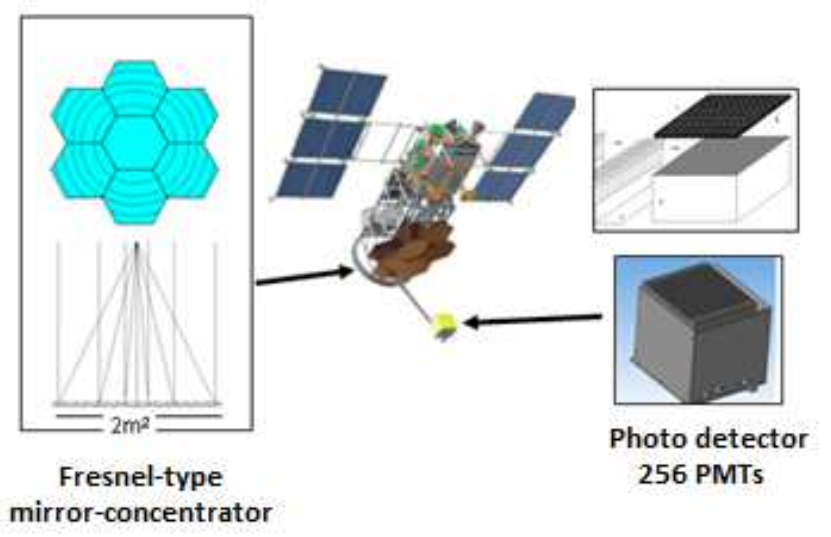

Fig.1. The main part of the UV telescope TUS installed on board "Lomonosov" satellite.

TUS is operating more than one year on board the Lomonosov satellite. The general design of TUS is determined by its main task of registering fluorescence and scattered (reflected) Cherenkov radiation of EASs in the UV band with a time resolution of $0.8 \mu \mathrm{s}$ in full temporal interval of 256 time steps. It can also be employed for measuring transient light events in the atmosphere with longer duration in modes with longer time samples implemented in the detector 
DAQ system. This design makes TUS a multi-purpose detector [21-23]. The energy threshold for TUS approximately equals $70 \mathrm{EeV}$, and the instrument will be able to register a few particles of UHECR above the threshold in 5 years of continuous operation assuming the energy spectrum obtained with the Auger installation for highly inclined EAS [24].

Preliminary results of observations of different natural phenomenon have been reported elsewhere: $[25,26,27]$. Here we present the main results concerning the observations of EAS UHECR candidates. They are as follows. TUS began measurements in space on May 19, 2016. The first months of work were mostly dedicated to testing hardware, software and various modes of operation. Continuous measurements were started in September 2016 with several gaps for the satellite technical service. By the end of May 2017, TUS has registered about 35,000 events at nocturnal parts of its orbit in the EAS mode with total exposure is approximately $530 \mathrm{~km}^{2} \mathrm{yr} \mathrm{sr}$. It is small in comparison with that of the largest ground-based arrays but sufficient to look for an UHECR above the TUS threshold energy [24]. In a search for an EAS, generated by an UHECR, it observes a large number of background events of various origin that take place in the nocturnal atmosphere of the Earth but they do not exclude a possibility to detect a true EAS events at energies above $70 \mathrm{EeV}$. More about the different types of the background transient events may be found in [22]. Such events are about $10 \%$ of the total number of measurements taken at moonless nights. As a result, 13 preliminary EAS candidates were selected and analyzed on the event by event basis according to the spatial-temporal criteria for horizontal and vertical EASs. Six of these candidates are eliminated from the further analysis because they do not meet the empirical criteria. Other 7 events are considered as horizontal (4 events) or vertical ( 3 events) EAS candidates. Fig. 2 demonstrates two types of candidates (values of the moving average of ADC counts calculated for 16 time samples for horizontal EAS candidate and 3 time samples for vertical are shown). The moving average curves in the figures are adjusted to have the same base level of the signals. The most interesting for the further analyses are four candidates for horizontal EAS (see Fig.2 left), which demonstrate a movement of the image in the FOV.
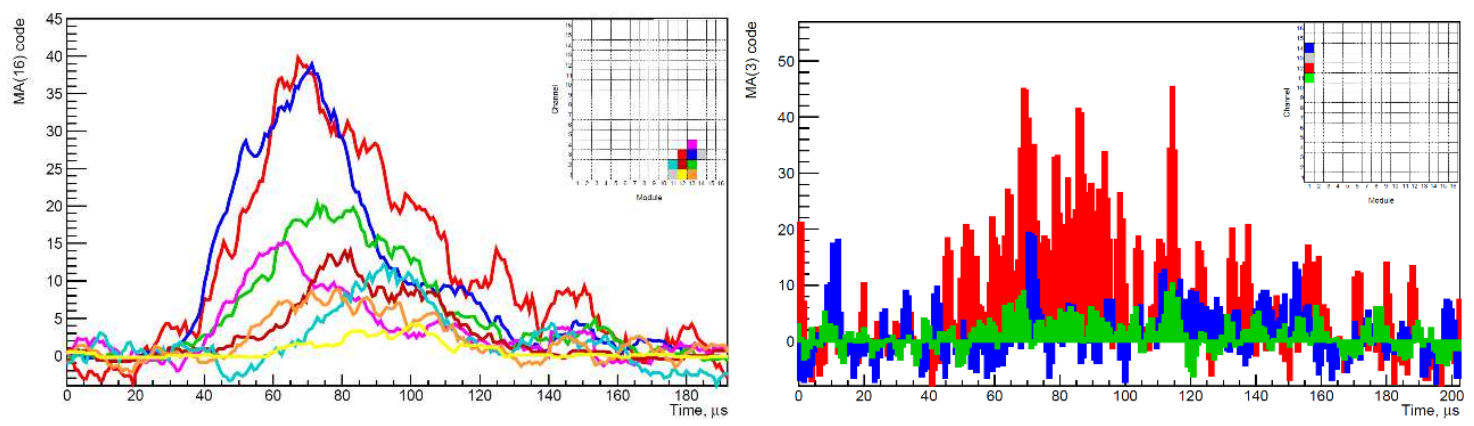

Fig.2. Two different types of candidates for EAS UHECR events horizontal (left) and vertical (right) (see for details [25]). Moving average of ADC counts for active channels and their pixel maps of events are shown.

Multi-level algorithm for the search of EAS-like events was developed in [26] and applied to the TUS data set analysis. As a result, at least one the best EAS candidate was selected and has analyzed. Selected events may contain some genuine EAS events at energy $\sim 100 \mathrm{EeV}$.

\section{GRB's observations results}

Lomonosov satellite payload includes the gamma-ray spectrometer named BDRG consisting of 3 orthogonally directed detector units and two wide field cameras named SHOK are placed in such a way that their fields FOV are overlapped by the GRB monitor detector's FOVs. The optical data can be stored by the trigger from gamma-detector. This allows multi-wavelength 
GRB observations in all-time scale of event evolution including optical light curves of prompt emission as well as of precursors. The real time data transfer to the GCN system (see for details [28]).

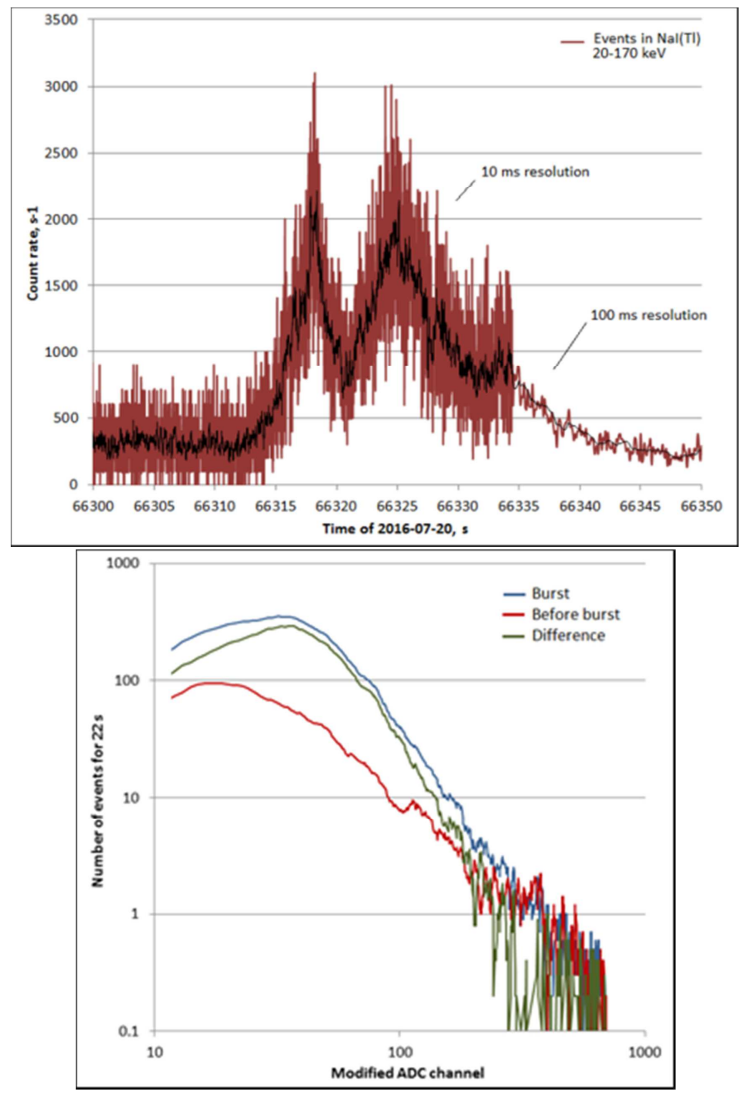

Fig.3. An examples of registration one of GRB event GRB 2016-07-20 in different temporal resolution: $10 \mathrm{msec}$ and $100 \mathrm{~ms}$ (left) and variations of energy spectra.

For one of events - GRB 161017 it was possible to compare "Lomonosov", Swift satellites data and on ground robotic MASTER - net observations [28] of optical emissions. The combined light curve of from "Lomonosov", MASTER-net and Swift observations is presented at Fig. 4. 


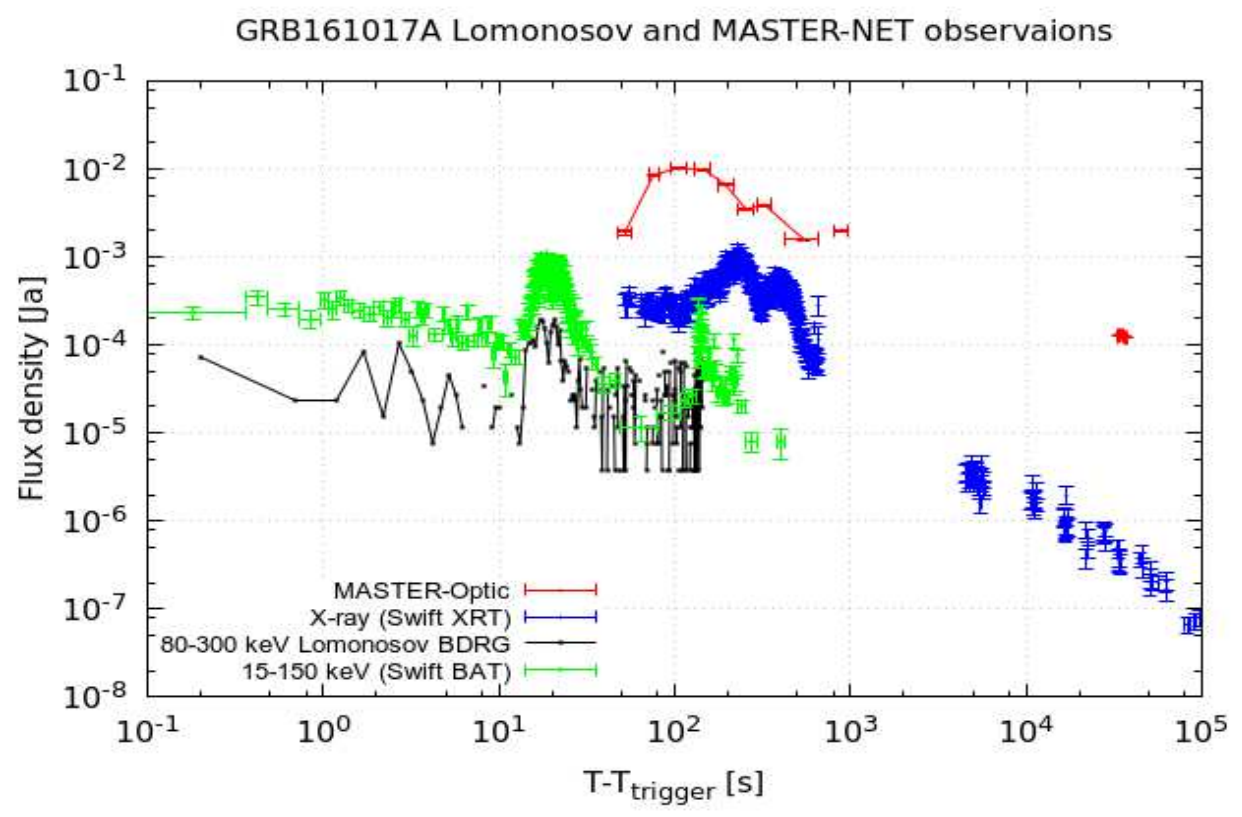

Fig. 4. Lomonosov, MASTER-net and Swift observations of GRB 161017A in broad bands:in optics and in gamma-rays [28].

It is known from several previous observations that prompt optical emission detected during the first few minutes after the onset of a GRB and in some events correlates with the prompt $\gamma$ ray emission, and in other cases early optical light curve is uncorrelated with the $\gamma$-ray light curve [29]. For this particular case of GRB 161017A it has been shown that optical emission is uncorrelated with $\mathrm{x}$ - and gamma - rays. Both $\mathrm{x}$ - and gamma-rays light curves have a similar structure, but optical emission rise up during more less quite time from $\sim 50$ till $\sim 120 \mathrm{~s}$ and even decay during second activity episode after $\sim 130 \mathrm{~s}$. Further search of this event in progress.

The third GRB instrument on board Lomonosov satellite named UFFO (Ultra-Fast Flash Observatory) is designed as a combination of a coded-mask hard X-ray telescope UBAT (Burst Alert and Trigger Telescope) and an optical telescope Slewing Mirror Telescope (SMT) with moving mirror that can be pointed to the GRB source for $\sim 1 \mathrm{~s}$ (Fig.5). Its characteristics, operation modes and first results will be discussed in separate papers [30, 31]. This allows the simultaneous GRB observations in gammas and optics in all-time scale of event evolution including obtaining optical light curves of prompt emission as well as of precursors. The SMT is the first orbital instrument, using motorized mirror for observations of the space, designed to shed light on first seconds of GRBs prompt UV/optical emission. The SMT is main component of the UFFO. Once GRB is detected by UFFO X-ray coded mask camera, SMT turns its motorized mirror within seconds to observe optical and UV emission of GRB. 


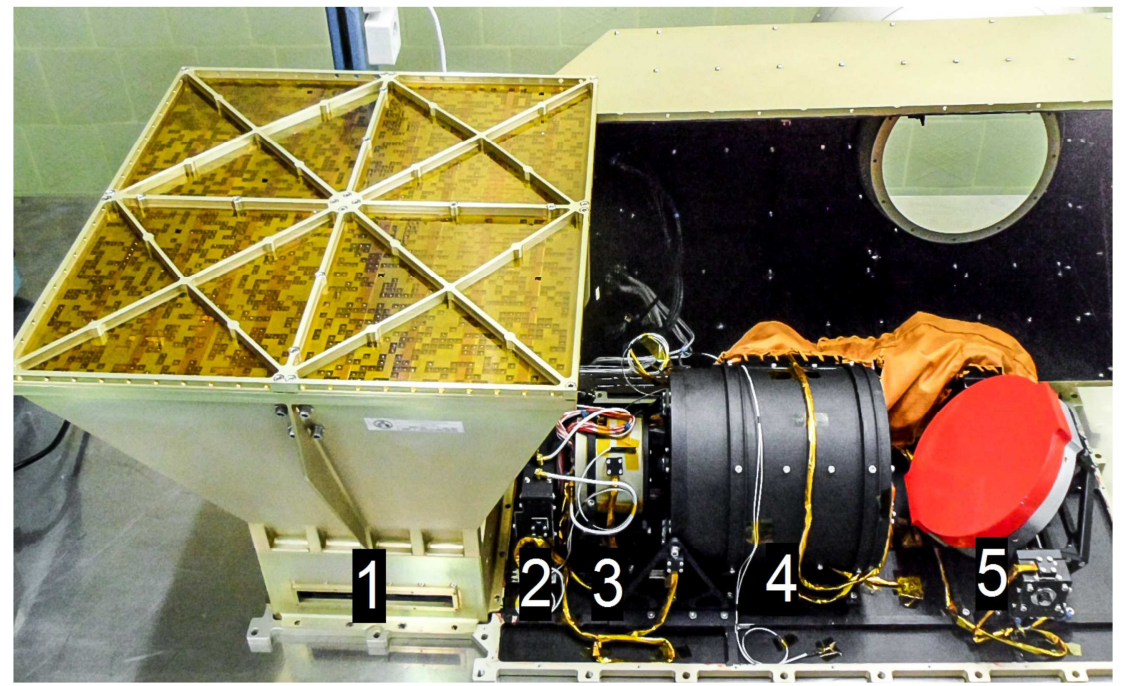

Fig. 5: Ultra-Fast Flash Observatory Pathfinder. (1) - UFFO-pathfinder; UBAT- X-ray coded musk camera, used for trigger and localization of GRB; SMT : (2) - Readout electronics; (3) - Intensified charge-coupled device detector (ICCD); (4) - $10 \mathrm{~cm}$ RC Telescope; (5) - Slewing mirror system (the mirror is under a red protection cover before launch).

After the some problems overcome, the SMT has been calibrated by the in-situ method. As for now, the UFFO-pathfinder is operating in orbit and the SMT is ready to observe GRB.

UBAT also has been checked and some results are presenting on Fig.6. Here is the correlation map from offline analysis where data taken for $150 \mathrm{~s}$ corresponding to the movement of $\approx 7^{\circ}$. This map is $\approx 70^{\circ} \times 70^{\circ} \mathrm{FOV}$. Analysis of this data in the progress, but preliminary description of this image is combination of particles hits and part of galactic plan's objects.

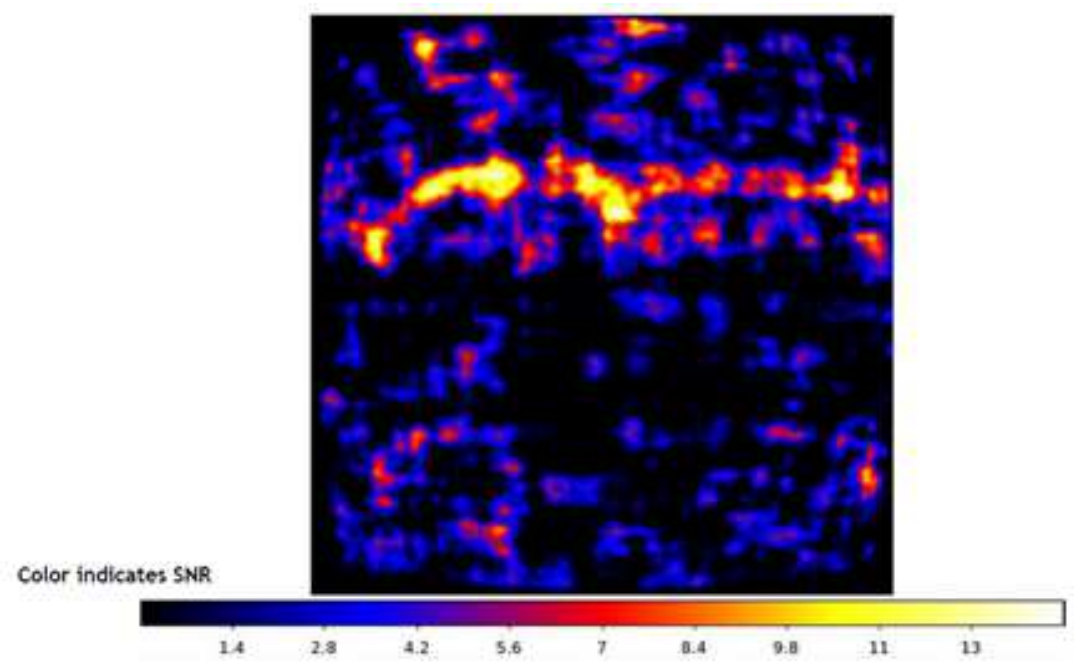

Fig.6. UBAT- X-ray coded musk camera: one of the first images.

\section{Conclusions}

Astrophysical space observatory "Lomonosov" for more than a year in operation. In the field of Astroparticle science studies there are search of two relevant astrophysical phenomena UHECR and GRB are in progress now. The first results within a year of Lomonosov's flight 
have been demonstrated: 1) the first year of TUS operation definitely show the possibility of registration of UHECR events from space that is - the possibility of selection and measurements of parameters of EAS UHECR candidates against numerous UV transient phenomena of natural and anthropogenic origin in the Earth's atmosphere; 2) effective registration of GRB events with high temporal and energy resolution. Also for the first time we presents the results of simultaneous measurements of GRB in space and their optical observations using the system of robotic on ground telescopes.

\section{References}

[1] V. Prosin et al. EPJ Web of Conferences 121, 03004 (2016)

[2] G. T. Zatsepin and V. A. Kuzmin, JETP Lett. 4, 78 (1966)

[3] K. Greisen. Phys. Rev. Lett., 16, 748 (1966)

[4] A. Aab, P. Abreu, M. Aglietta, et al., Astrophys. J., 804:15 (2015).

[5] R. Abbasi et al., arXiv:1503.07540 [astro-ph.HE]

[6] R. U. Abbasi et al., Astrophys. J. 790, L21 (2014) [arXiv:1404.5890 [astro-ph.HE]

[7] A. Aab et al., Astrophys. J. 804, no. 1, 15 (2015), arXiv:1411.6111 [astro-ph.HE]

[8] R. Benson and J. Linsley., In Bulletin of the American Astronomical Society, 12, 818 (1980)

[9] R. Benson and J. Linsley. 17th ICRC proceedings, 8, 145(1981)

[10] R. Strietmatter et al. AIP Conf. Proceedings (Woodbury, N.Y.) 433, 95 (1998)

[11] L.Scarsi et al. Proposal to ESA F2/F3 misions (2000)

[12] Adams, J.H., S. Ahmad, J.-N. Albert, et al. Experimental Astronomy, 40(1):3, (2015).

[13] Adams, J.H., S. Ahmad, J.-N. Albert, et al. Experimental Astronomy, 40(1):19-44, 2015.

[14] M. I. Panasyuk, M. Casolino, G. K. Garipov, et al. Journal of Physics Conference

Series, 632(1):012097, (2015)

[15] K. Hurley. Nature 357: 112. (1992)

[16] Fishman, C.J. and Meegan, C.A. Annual Review of Astronomy and Astrophysics 33: 415-458. doi:10.1146/annurev.aa.33.090195.002215 (1995).

[17] V. V. Alexandrov et al., ICRC Proceedings , 2:831 (2001)

[18] B. A. Khrenov et al., in H. Salazar, L. Villasenor, and A. Zepeda, editors, Observing Ultrahigh Energy Cosmic Rays from Space and Earth, 566, 57, (2001)

[19] M. Casolino, et al., 35 ${ }^{\text {th }}$ ICRC, Proceedings of science (this issue) (2017).

[20] P. A. Klimov et al. ArXiv,1706.04976 [astro-ph.IM] (2017)

[21]M. Zotov et al., ArXiv,1703.09484, [astro-ph.IM] (2017)

[22] B. A. Khrenov et al., ArXiv, 1704.07704 [astro-ph.IM] (2017).

[23] A. A. Grinyuk et al., Journal of Physics Conference, Series, 409(1):012105 (2013)

[24] The Pierre Augur collaboration. J.1 of Cosmology and Astroparticle Physics,2015(08):049 (2015)

[25] S.V. Biktemerova et al., ArXiv:1706.05369v1 [astro-ph.IM] (2017)

[26] L. Tkachev et al., $35^{\text {th }}$ ICRC, Proceedings of science (this issue) 2017.

[27] P.Klimov et al, $35^{\text {th }}$ ICRC, Proceedings of science (this issue) (2017)

[28] V. Bogomolov et al., $35^{\text {th }}$ ICRC, Proceedings of science (this issue) (2017)

[29] E.S. Gorbovskoy et al., MNRAS 421, 1874 (2012)

[30] G. Gaikov et al., $35^{\text {th }}$ ICRC, Proceedings of science (this issue) (2017)

[31] H.Jeong et al., $35^{\text {th }}$ ICRC, Proceedings of science (this issue) (2017)

\section{The list of Lomonosov - UHECR/TLE \& GRB collaborations}

V. Sadovnichy ${ }^{1}$, M.Panasyuk ${ }^{2,3}$, S. Svertilov ${ }^{2,3}$, V.Bogomolov ${ }^{2,3}$, A.Amelushkin ${ }^{2}$, V. Barinova $^{2}$, A. Bogomolov ${ }^{2}$, A. Iyudin ${ }^{2}$, V. Kalegaev ${ }^{2}$, E. Kuznetsova ${ }^{2,3}$, I.Myagkova ${ }^{2}$, A. Minaev $^{2}$, Min Nguyen ${ }^{2}$, V. Petrov ${ }^{2}$, I. Yashin ${ }^{2}$, V. Lipunov ${ }^{4}$, E.Gorbovskoy ${ }^{4}, \mathrm{~N}$. Chirskaya $^{2}$, V. Eremeeva ${ }^{2}$, G. Garipov ${ }^{2}$, N. Kalmykov ${ }^{2}$, M.Kaznacheeva ${ }^{2,3}$, P. Klimov², M.Lavrova ${ }^{2}$, V. Petrov ${ }^{2}$, S. Sharakin ${ }^{2}$, A. Shirokov ${ }^{2}$, B. Khrenov ${ }^{2}$, I. Yashin ${ }^{2}$, M. Zotov $^{2}$,I. Park ${ }^{5}$, S. Jeong ${ }^{5}$, S. Jeong, M. Kim ${ }^{5}$, J. Lee ${ }^{5}$, A.Castro-Tirado ${ }^{6}$, A. Botvinko ${ }^{7}$, 
A. Puchkov ${ }^{7}$, O.Saprykin $^{7}$, A.Senkovsky ${ }^{7}$, S.Biktemerova $^{8}$, L. Tkachev $^{8,9}$, V. Grebenyuk $^{8,9}$, A. Grinyuk ${ }^{8}$, A.Tkachenko ${ }^{8}$, O. Martinez ${ }^{10}$, E. Ponce ${ }^{10}$, H. Salazar ${ }^{10}$.

${ }^{1}$ Lomonosov Moscow State University, GSP-1, Leninskie Gory, Moscow, 119991, Russia

${ }^{2}$ Skobeltsyn Institute of Nuclear Physics of Lomonosov Moscow State University, GSP1, Leninskie Gory, Moscow, 119991, Russia

${ }^{3}$ Physics department of Lomonosov Moscow State University, GSP-1, Leninskie Gory, Moscow, 119991,Russia

${ }^{4}$ Sternberg Astronomical Institute of Lomonosov Moscow State University, GSP-1, Leninskie Gory, Moscow, 119991,Russia

5 Department of Physics and ISTS, Sungkyunkwan University, Seobu-ro 2066, Suwon,440-746 Korea

${ }^{6}$ Instituto de Astrofisica de Andalucia (IAA-CSIC),P.O.Box 03004, E-18080, Granada, Spain

${ }^{7}$ Space Regatta Consortium, ul. Lenina, 4a, 141070 Korolev, Moscow region, Russia

${ }^{8}$ Joint Institute for Nuclear Research, Joliot-Curie, 6, Dubna, Moscow region, Russia, 141980

${ }^{9}$ Dubna State University, University str., 19, Bld.1, Dubna, Moscow region, Russia

${ }^{10}$ Benemerita Universidad Autonoma de Puebla, 4 sur 104 Centro Historico C.P.72000, Puebla, Mexico 